\title{
Asociación de firutales de limón persa (Citrus latifolia) y pallima de coco (Cocos nucifera L.) con el aporte de materia seca por cultivos anuales
}

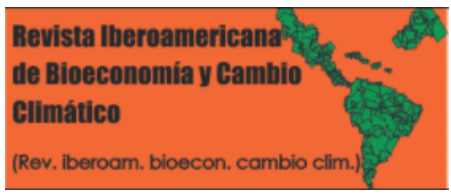

\author{
Association of Persian lemon fruit trees (Citrus latifolia) and \\ coconut palm (Cocos nucifera L.) with the contribution of dry \\ matter by annual crops
}

\author{
Rebolledo-Martínez, Laureano; Megchún-García, Juan Valente; \\ Rebolledo-Martínez, Andrés; Orozco-Corona, Dulce María; Editor \\ Académico Prof. Ph.D Carlos Alberto Zúniga-González
}

Laureano Rebolledo-Martínez rebolledo.laureano@inifap.gob.mx

Instituto Nacional de Investigaciones Forestales, Agrícolas y Pecuarias, México

Juan Valente Megchún-García

juanmg_3@hotmail.com

Universidad Veracruzana., México

Andrés Rebolledo-Martínez

rebolledo.laureano@inifap.gob.mx

Instituto Nacional de Investigaciones Forestales,

Agrícolas y Pecuarias, México

Dulce María Orozco-Corona

Unidad Académica de Estudios Nucleares de la

Universidad Autónoma de Zacatecas., México

Editor Académico Prof. Ph.D Carlos Alberto

Zúniga-González

Universidad Nacional Autónoma de Nicaragua,

León. Escuela de Ciencias Agrarias y Veterinaria.

Departamento de Agroecologia., Nicaragua

Revista Iberoamericana de Bioeconomía y Cambio Climático

Universidad Nacional Autónoma de Nicaragua, León, Nicaragua ISSN-e: 2410-7980

Periodicidad: Semestral

vol. 5, núm. 10, 2019

czuniga@ev.unanleon.edu.ni

Recepción: 15 Julio 2019

Aprobación: 04 Diciembre 2019

URL: http://portal.amelica.org/ameli,

jatsRepo/394/3941757003/index.html

DOI: https://doi.org/10.5377/ribcc.v5i10.8968

Autor de correspondencia: juanmg_3@hotmail.com
Resumen: La asociación de limón persa y cocotero con cultivos anuales contribuye a la mejora de las características de los suelos principalmente en el contenido de materia orgánica al incorporar los residuos de cosecha con un manejo integral. El objetivo es implementar un sistema de cultivos de maíz y frijol intercalados con frutales de limón persa y palma de coco. El trabajo se estableció en octubre de 2017, en el Hatillo, municipio de la Antigua, Veracruz, con árboles de frutales de limón persa y palma de coco, en cepas con la adición de abonos orgánicos. Antes del establecimiento se abonó con 18 t.ha-1 de materia seca de la caña de azúcar y posteriormente se establecieron los cultivos de frijol Michigan, negro Veracruz y maíz H-520, estos cultivos fueron manejados en diferentes fechas de siembra durante el periodo 2018 y 2019. Se midieron en los frutales, altura de planta, diámetro de tallo, número de hojas y ramas, aporte de materia seca al suelo y se realizaron análisis de suelo en árboles de limón persa, palma de coco y cultivos anuales. Los resultados para limón persa presentan diferencias significativas a los 386 días, con respecto al diámetro de tallo, altura de planta, número de ramas. Con respecto a la palma de coco, el diámetro de tallo presentó diferencias significativas a partir de los 190 días, en la altura de la palma los valores fueron diferentes a los 386 días. En el aporte de materia seca el frijol negro Veracruz, aportó mayor cantidad al suelo con 8,426 t/ha; el maíz híbrido H-520 aportó mayor cantidad de materia seca en la fecha del 23 de febrero del 2018 con 11,333 t/ha. Al analizar la materia orgánica del suelo, se encontró mayor porcentaje en el sistema cultivos intercalados, superando al monocultivo de caña de azúcar. La asociación de especies permite mejorar las condiciones agroecológicas en el manejo de los cultivos.

Palabras clave: Intercalados, Frutales, Materia orgánica.

Abstract: The association of Persa lemon and coconut with annual crops contributes to the improvement of soil characteristics mainly in organic matter content by incorporating crop residues with an integrated management. The objective is to implement a system of corn and bean crops interspersed with Persa lemon and coconut palm fruit 
trees. The work was established in October 2017, in Hatillo, municipality of Antigua, Veracruz, with fruit trees of Persa lemon and coconut palm, in vines with the addition of organic fertilizers. Before the establishment was fertilized with $18 \mathrm{t}$. ha-1 of dry matter from sugar cane and then established the crops of Michigan bean, black Veracruz and H-520 corn, these crops were managed at different planting dates during the period 2018 and 2019. They were measured in fruit trees, plant height, stem diameter, number of leaves and branches, contribution of dry matter to the soil and soil analysis were performed on Persa lemon trees, coconut palm and annual crops. The results for Persa lemon show significant differences at 386 days, with respect to stem diameter, plant height, number of branches. With respect to the coconut palm, the diameter of stem presented significant differences from the 190 days, in the height of the palm the values were different to the 386 days. In the contribution of dry matter the black bean Veracruz, contributed greater amount to the soil with 8426 t/ha; the hybrid corn $\mathrm{H}-520$ contributed greater amount of dry matter in the date of February 23, 2018 with $11333 \mathrm{t} / \mathrm{ha}$. When analyzing the organic matter of the soil, a higher percentage was found in the intercropping system, surpassing the monoculture of sugar cane. The association of species allows to improve the agro ecological conditions in the management of the crops.

Keywords: Crop rotation, Fruit Trees, Organic Matter.

\section{INTRODUCCIÓN}

La agricultura mexicana está siendo afectada por el Tratado de Libre Comercio (TLC), con América del norte, entre los cultivos más afectados es el maíz (Zea mays L.) por la competencia con existe con Estados Unidos. Esta situación implica generar nuevas alternativas para mejorar la bioeconomia de la producción campesina, entre ellos el 80\% de la producción de maíz en México destinada para el consumo humano, ahora se reserva para la producción de forrajes y para la eco-intensificación de la producción animal (Ortega et al., 2005). Considerando que la producción es condicionada por las grandes agroindustrias transnacionales, que dominan las tecnologías generando el avance de una agricultura sin agricultores, con el uso de prácticas y métodos asociados a la expansión de la producción de los cultivos transgénicos (Paz et al., 2013).

Es necesario buscar nuevas alternativas de producción que permitan mejorar las condiciones agroecológicas en los sistemas de producción. Por otra parte, el concepto de la bioeconomía en la agricultura, surge a través del uso de materia orgánica o vegetal de desecho como materia prima para la generación de energía y combustibles; sin embargo, se han venido añadiendo otros usos a los desechos de la agricultura, que es destinada a la generación de bioinsumos, bioles u otros materiales de combustión, por lo que se propone como estrategia la modalidad de una producción diversificada a través de cultivos intercalados (Mercados, 2016).

La asociación de cultivos tiene diversas modalidades, dependiendo el tipo de explotación, necesidades, área del cultivo. Estos puede ser como cultivos múltiples intercalados, mezclados intercalados, intercalados en hileras, intercalados en franjas, en su mayoría esto sistemas dependerán del tipo de especies a cultivar y las

Notas DE AUTOR

juanmg_3@hotmail.com 
necesidades del mercado (Megchún, 2007). La asociación es la utilización de dos o más especies diferentes en forma simultánea y sirve para una mejor utilización del terreno, menor riesgo de pérdida por plagas y enfermedades en una de las especies, la forma más común es la asociación de frijol y maíz entre los meses de mayo y junio en forma simultanea (Godoy et al., 2011). La asociación de los cultivos permite proteger el suelo de la erosión y reducir las labores culturales para el control de malezas, mejorar la fertilidad del suelo y diversificar la producción mejorando las condiciones bioeconomicas de los productores (Duarte et al., 1994). En este sentido los agroecosistemas son extremadamente simplificados y frágiles, con una progresiva pérdida de la agrodiversidad tanto en el sentido espacial, territorial y temporal (Leguía et al., 2004).

destinada para el consumo en fresco de limón persa, y el resto de la producción es enviada a la industria para la extracción de subproductos (Hernández et al., 2017). Brasil es el principal productor de cocotero en el mundo, el cocotero necesita grandes cantidades de nutrientes para el desarrollo de sus órganos como frutos, raíces y engrosamiento del tronco, por lo que extrae grandes cantidades de nitrógeno y potasio (Matías et al., 2008). La incorporación de materia seca es una alternativa para mejorar las condiciones nutrimentales de los suelos donde se establece este frutal. El cocotero es considerado una joya de los trópicos y uno de los cultivos más importantes en el mundo, de las cuales se estima que existen 3000 millones de hectáreas cultivadas, en México existen dos tipos de cocoteros en términos generales, el tipo silvestre "niu kafa" y el tipo domesticado "niu val" distribuidos en la costa del atlántico y pacífico, además que existen más de 100 productos que se elaboran de la palma de coco (Granados y López, 2002).

La asociación de cultivos básicos con frutales de cocotero y limón persa permite mejorar la rentabilidad y productividad de los cultivos y no depender del monocultivo, por lo que establecer cultivos asociados en forma escalonada de árboles de cocotero y limón persa, permite obtener alimentos en diferentes fechas de siembra para el autoconsumo (Rebolledo et al., 2018). Con el establecimiento de cocotero intercalado con limón y frijol en un sistema agroforestal en un periodo de tres años, se pude obtener rendimientos de frijol, y se inicia la producción de limón y un año después es posible iniciar con la cosecha de fruta de coco (Balderas y González, 2013). El objetivo de esta investigación es implementar un sistema de cultivos de maíz y frijol intercalados con frutales de limón persa y palma de coco manejando para conocer el efecto y producción de materia seca al suelo.

\section{Materiales Y MÉTODOS}

El experimento se estableció en la localidad del Hatillo, municipio de la Antigua Veracruz, ubicado en las coordenadas $19^{\circ} 16^{\prime} 58^{\prime \prime}$ de latitud norte y $96^{\circ} 18^{\prime} 26^{\prime \prime}$ longitud oeste, altitud de 41 metros. Figura 1 


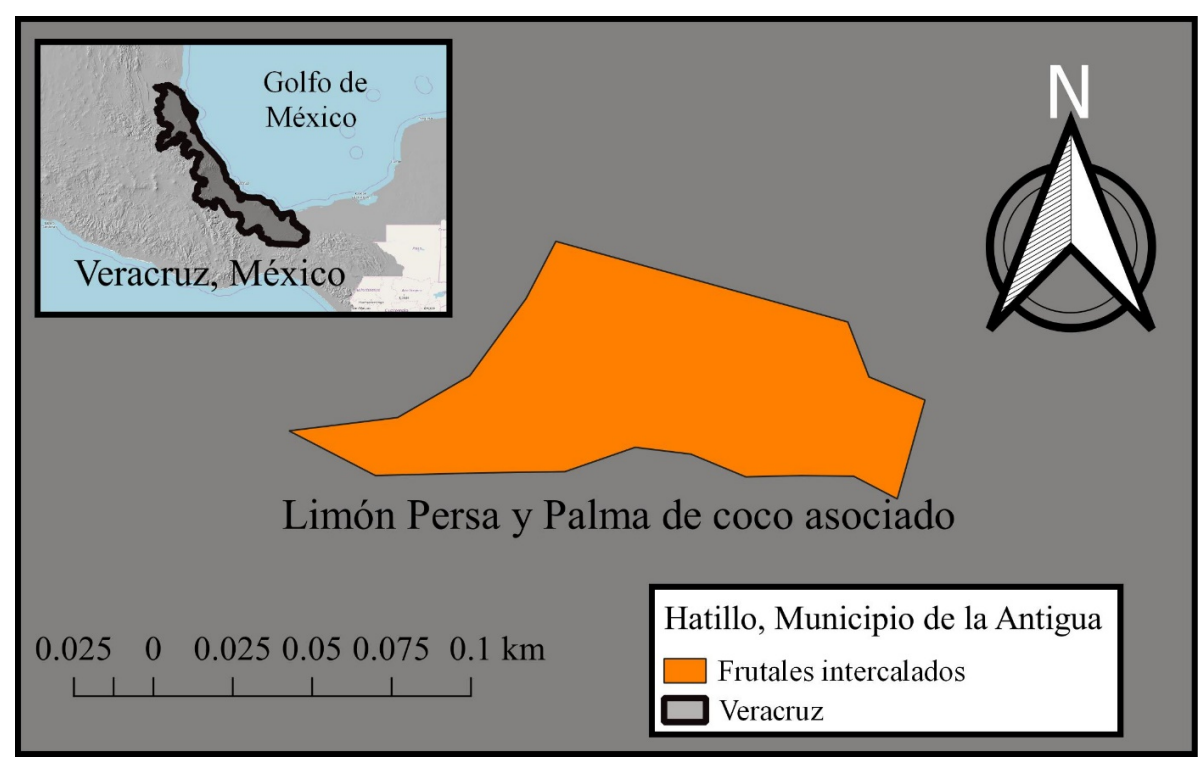

FIGURA 1.

Ubicación de la huerta de limón Persa intercalado con palma de coco en asociación con cultivos anuales.

\section{MÉTOdo}

En abril de 2017 el trabajo se inició en un terreno con textura de franco arenosa con una precipitación de $1000 \mathrm{~mm}$, se realizó el aporte de 18 toneladas de materia seca por hectárea obtenida de la cosecha de la caña de azúcar (Saccharum officinarum L.). Durante su desarrollo se manejó un sistema de riego por goteo con cintillas. En octubre de 2017, se plantaron especies de palma de coco (Cocos nucifera L.) en cepas de $40 \mathrm{x}$ 40 x $50 \mathrm{~cm}$ de profundidad, adicionando dos kg de materia seca al fondo de la cepa, seguido de una capa de suelo de cinco $\mathrm{cm}$. El arreglo espacial fue de 4 hileras establecidas en área de 2,500 m2, la distancia entre hileras fue de $7 \mathrm{~m}$, y la distancia entre árboles de coco y limón fue de $3.5 \mathrm{~m}$. En enero de 2018, se establecieron árboles de limón persa (Citrus latifolia Tan) en cepas de $30 \times 30 \times 40 \mathrm{~cm}$ de profundidad adicionando un $1 \mathrm{~kg}$ de materia seca al fondo seguido de una capa de suelo de cinco $\mathrm{cm}$. Entre las hileras de los árboles en enero y febrero se sembró en diferentes fechas de siembra maíz (Zea mays L.) y frijol (Phaseolus vulgaris L.) ocupando una superficie de $210 \mathrm{~m} 2$.

El cultivo de frijol intercalado se estableció el 30 de enero de 2018 con la variedad Michigan y en el 2019 la variedad Negro Veracruz, el arreglo topológico fue de $40 \mathrm{~cm}$ entre hileras y $10 \mathrm{~cm}$ entre plantas. Para el cultivo de maíz intercalado se utilizó el híbrido H-520, con diferentes fechas de siembra, estos corresponden al 26 de enero de 2018, 19 de febrero de 2018, 23 de febrero del 2018 y el 16 de febrero del 2019, con una densidad de siembra de 50,000 plantas ha-1. Sembrados una semilla cada $25 \mathrm{~cm}$. El manejo de las especies cultivadas en este sistema consistió en el uso de bioestimulante de plantas y humo líquido que actúan como repelente de plagas, para la nutrición se utilizó abono tipo bocashi, composta, MM líquido y sulfocalcio, elaborados de manera artesanal.

Entre las hileras de los frutales el frijol y el maíz intercalado, se midió el aporte de materia seca al suelo durante el periodo del 2018 al 2019. Las variables de crecimiento y desarrollo en los frutales, fueron el diámetro de tallo, altura del árbol, número de ramas en el limón persa, número de hojas en la palma de coco y frutos producidos por árbol de limón persa.

Para conocer el contenido de materia orgánica se tomaron muestras compuestas del suelo a una profundidad de 0 a $20 \mathrm{~cm}$ y 20 a $40 \mathrm{~cm}$, la muestra compuesta se formó de tres submuestras tomadas en el área de la copa de árbol, de acuerdo a los siguientes tratamientos. 1. Limón persa con aporte de materia seca, 
2. Limón persa sin aporte de materia seca, 3. Palma de coco con aporte de materia seca, 4. Palma de coco sin aporte de materia seca, 5 . El testigo correspondió al monocultivo de caña de azúcar.

El pH y el contenido de N, P, K, Ca, Mg, Fe, Cu, Zn y Mn fueron medidos en muestras de suelo obtenidos de los siguientes tratamientos: 1 . Asociación de frijol y maíz $\left(\mathrm{NO}_{3}\right)$, 2. Limón sin materia seca, 3. Limón con materia seca, 4. Palma sin materia seca, 5. Palma con materia seca y 6. Monocultivo de Caña de azúcar.

Las muestras fueron analizadas con base a las siguientes normas mexicanas en las instalaciones del Campo Experimental Cotaxtla, Veracruz, la textura de las muestras analizadas correspondieron a un suelo migajón arcilloso, de acuerdo al diagrama triangular (USDA, 2019), Cuadro 1.

\section{CUADRO 1}

Normas mexicanas utilizadas para las determinaciones físicoquímicas en suelo de árboles con y sin el uso de materia seca.

\begin{tabular}{|c|c|}
\hline Normas Mexicanas para Suelo & $\begin{array}{l}\text { Determinaciones físicas y } \\
\text { químicas en suelo }\end{array}$ \\
\hline $\begin{array}{l}\text { NOM } 021 \text { RECNAT } 2000 \\
\text { AS-09 }\end{array}$ & Textura del suelo \\
\hline 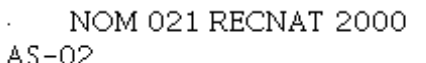 & $\mathrm{pH}$ \\
\hline $\begin{array}{l}\text { NOM } 021 \text { RECNAT } 2000 \\
\text { AS-07 }\end{array}$ & Materia orgánica \\
\hline $\begin{array}{l}\text { NOM } 021 \text { RECNAT } 2000 \\
\text { AS-08 }\end{array}$ & $\begin{array}{l}\text { Nitrógeno inorgánico } \\
\text { (NH4+NO3) }\end{array}$ \\
\hline $\begin{array}{l}\text { NOM } 021 \text { RECNAT } 2000 \\
\text { AS-11 }\end{array}$ & Fósforo \\
\hline $\begin{array}{l}\text { NOM } 021 \text { RECNAT } 2000 \\
A-12\end{array}$ & Potasio \\
\hline $\begin{array}{l}\text { NOM } 021 \text { RECNAT } 2000 \\
\text { AS }-12\end{array}$ & Calcio \\
\hline $\begin{array}{l}\text { NOM } 021 \text { RECNAT } 2000 \\
\text { AS }-12\end{array}$ & Magnesio \\
\hline $\begin{array}{l}\text { NOM } 021 \text { RECNAT } 2000 \\
\text { AS-14 }\end{array}$ & Fierro \\
\hline $\begin{array}{l}\text { NOM } 021 \text { RECNAT } 2000 \\
\text { AS-14 }\end{array}$ & Cobre \\
\hline $\begin{array}{l}\text { NOM } 021 \text { RECNAT } 2000 \\
\text { AS-14 }\end{array}$ & Zinc \\
\hline $\begin{array}{l}\text { NOM } 021 \text { RECNAT } 2000 \\
\text { AS- } 14\end{array}$ & Manganeso \\
\hline
\end{tabular}

Nota La información de los datos fueron analizados con el software Minitad versión 17, el cual correspondió a un análisis de varianza al 95\% de Tukey.

\section{Resultados y Discusión}

Los resultados de la Figura 2, muestran diferencias estadísticas significativas para limón persa en la variable diámetro de tallo a los 386 días de edad, al compararlos al momento de su establecimiento en campo y a los 190 días; en la altura del árbol de limón, manejados con materia seca se observó diferencias estadísticas significativas a los 386 días de edad. Trabajos similares en Martínez de la Torre, Veracruz, en arboles de limón persa de cuatro años de edad, con patrones de citrumelo de Swingle, reportan el desarrollo de diámetros de $19.23 \mathrm{~cm}$ superando a los portainjertos Carrizo, Rugoso y Volkameriano, plantados en suelos de textura arcillosa (Curti et al., 2012). 

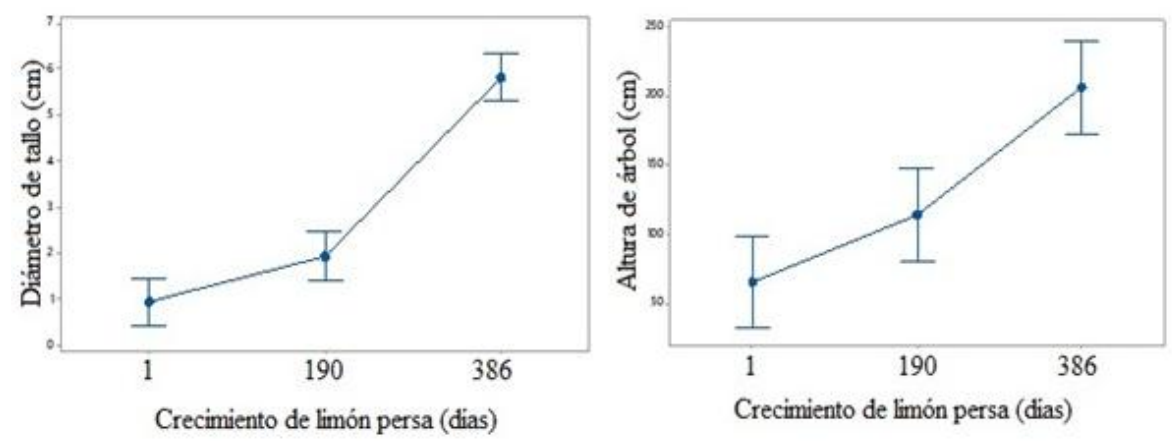

FIGURA 2.

Diámetro de tallo y altura de árbol del limón persa

En la Figura 3, se presenta el crecimiento del árbol de limón Persa con el aporte de materia seca, el cual propició el desarrollo de ramas en los arboles presentándose diferencias estadísticas significativas a los 190 días y 386 días de edad. Con respecto a la producción de frutos de limón se obtuvo diferencias estadísticas significativas al analizar las interacciones a la edad de los árboles de 386 días con el primer día de plantado, pero al comparar la edad del árbol a los 190 días con la edad de 386 días se observó diferencias significativas en la producción de limón persa. Es necesario que los árboles de limón persa se poden cuando se establecen en altas densidades de plantación debido a que no se sugiere que la altura del árbol exceda el ancho de las calles, la poda permite la intercepción de luz en la copa del árbol (Curti et al., 2012).
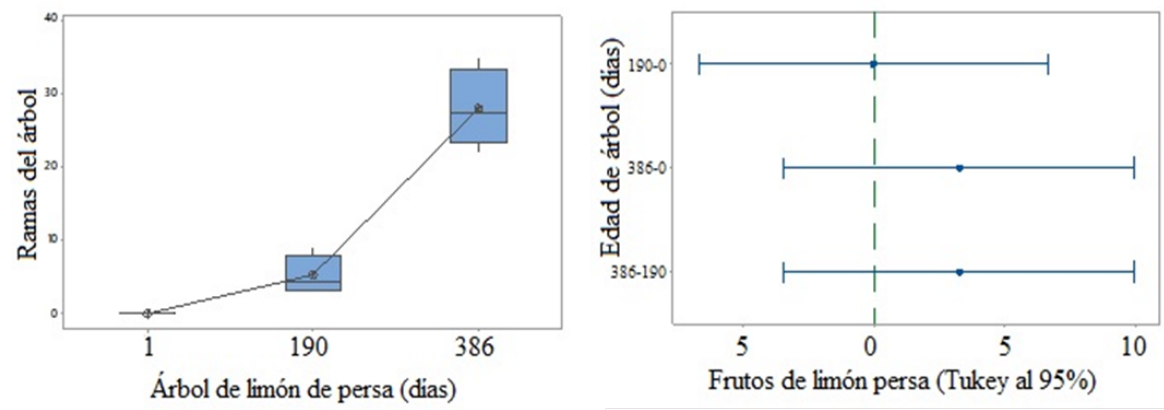

FIGURA 3

Desarrollo de ramas y frutos de limón persa

En las palmas de cocos manejados con el aporte de materia seca, los resultados presentados en la Figura 4, sobre el diámetro de tallo se observan diferencias estadísticas a los 190 y a los 386 días de establecido con incrementos del 133.3 y del $142.8 \%$. Con respecto a la altura de la palma de coco, se presenta incrementos significativos del $106.2 \%$ a partir de los 190 días de edad presentando diferencias estadísticas significativas al 95\% de confiabilidad. Los requerimientos nutrimentales de la palma de coco corresponden a la edad del árbol, observándose un incremento en las primeras etapas de desarrollo; palmas de coco enano con tres años de edad alcanzaron crecimientos inferiores a $5.2 \mathrm{~m}$ de altura manejados con diferentes dosis de nitrógeno por año (Matias et al., 2019). 

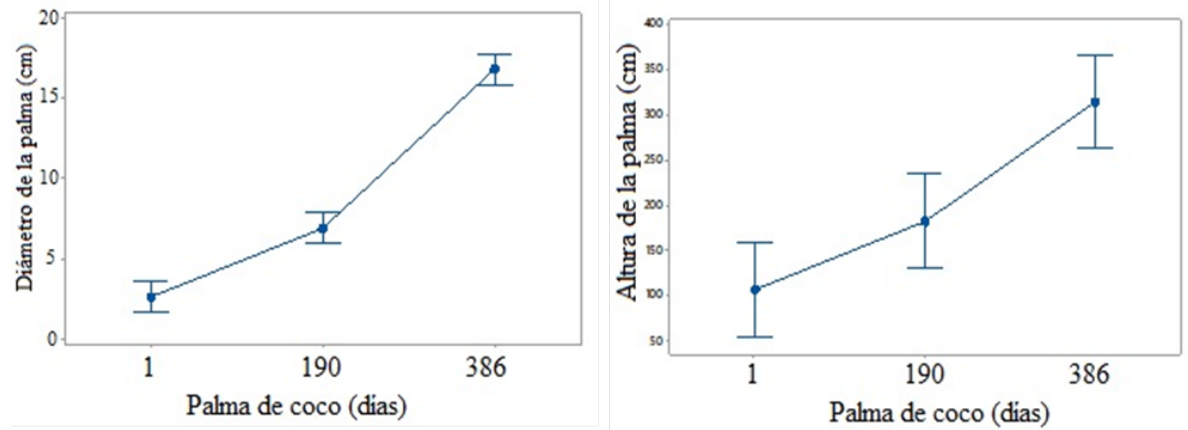

FIGURA 4.

Diámetro de tallo y altura de la palma de coco

Con respecto a la producción de hojas en la palma de coco, en las interacciones entre la edad de planta, se observó diferencias estadísticas significativas al comparar la edad del árbol de 386 días con el inicio de la plantación y cuando alcanzaron los 190 días (Figura 5). El desarrollo de las palmas de cocos va a ser influenciados por el contenido nutrimental del aporte de materia seca al suelo. Por lo que se espera que la producción de hojas incremente a temprana edad, existen valores medios de 22.9 hojas en palmas de coco enano a los tres años de edad, manejados con fertilizantes sintéticos (Matias et al., 2019). La importancia radica en el aprovechamiento de las hojas para el techado de viviendas y la fabricación de cestos, sombreros, cortinas, escobas (Granados y López, 2002).

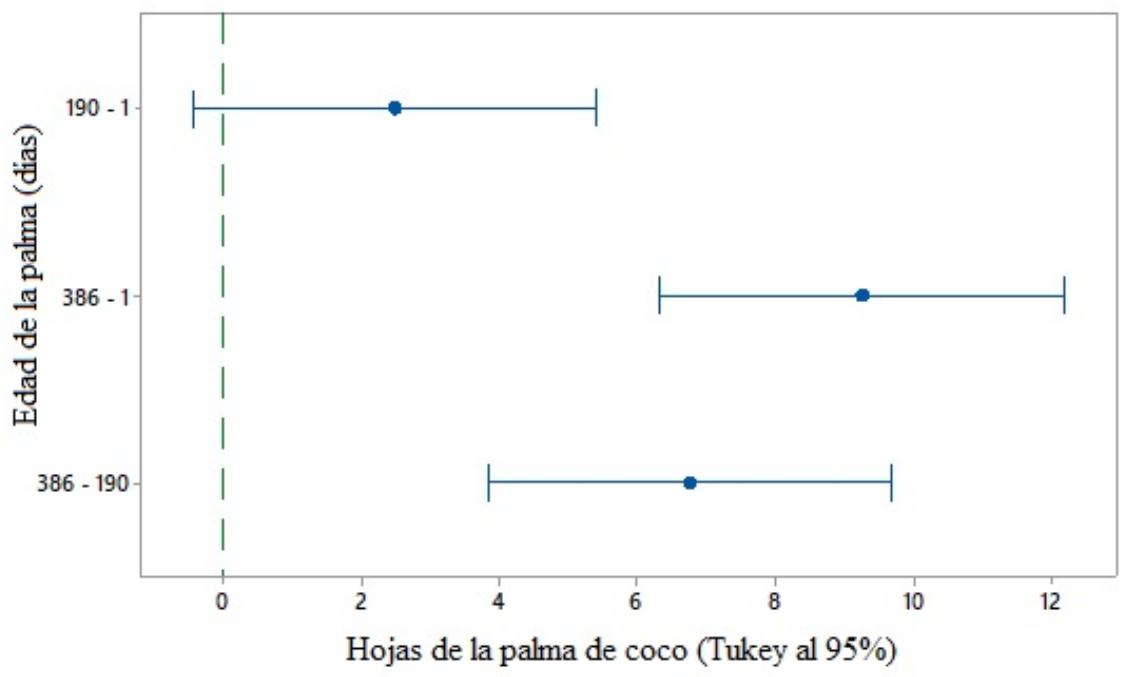

FIGURA 5.

Desarrollo de hojas en palma de coco

El cultivo de frijol asociado entre los árboles frutales de limón persa y cocotero durante el ciclo del 2018 al 2019 presentaron aportes significativos en el rendimiento de materia seca, se puede observar que la variedad negro Veracruz produjo $8.4 \mathrm{t} / \mathrm{ha}$ superiores estadísticamente significativo a las demás fechas de siembra, donde se asoció el cultivo de frijol con los frutales tropicales; esto pudo ser influenciado por la mejora de las condiciones del suelo, al incorporar la materia seca al suelo, debido a que el rendimiento de la producción de materia seca por ciclo de cultivo va en aumentando (Cuadro 2). Estudios sobre producción de materia seca en pastos mencionan que se incrementan significativamente del primero, al segundo y tercer año de producción, el cual estará relacionado con la especie y las condiciones climáticas que caracterizan el periodo de establecimiento del cultivo (Fernández et al., 2004). Con respecto a la asociación de maíz y frijol, existe 
evidencia de mejor desarrollo y rendimiento en el frijol asociado con maíz que en el sistema de monocultivo, para el caso del maíz y los componentes del rendimiento no fueron afectados al asociarlos con las variedades de frijol (Godoy et al., 2011).

CUADRO 2.

Aporte de materia seca del cultivo de frijol al suelo intercalado

\begin{tabular}{lll}
\hline Frijol & Año de siembra & $\begin{array}{l}\text { Aporte de materia de } \\
\text { seca (kg/ha) }\end{array}$ \\
\hline 1. Michigan & 2018 & $2,953 \mathrm{~b}$ \\
2. Michigan & 2019 & $5,556 \mathrm{ab}$ \\
3. Negro & 2019 & $8,426 \mathrm{a}$ * \\
Veracruz & \\
"Letras diferentes representa diferencias estadística \\
Significativa al 95\%.
\end{tabular}

Nota ${ }^{*}$ Letras diferentes representa diferencias estadística significativa al $95 \%$.

Con respecto al maíz híbrido $\mathrm{H}-520$ hay una variabilidad con respecto a la producción de materia seca, pero se logró obtener 11.3 t/ha en la fecha de siembra del 23 de febrero del 2018, siendo superior a las demás fechas de siembra, por lo que se supone que las características fisiológicas de las plantas, intervinieron en la sinterización de los nutrimentos que son traslocados para la producción de semillas (Cuadro 3). Aunque los rendimientos en producción de materia seca del hibrido H-520, son inferiores a los reportados en quince híbridos de maíz intermedios y precoces, se reportan rendimientos de materia seca entre los rangos de 18 a $22 \mathrm{t} /$ ha y de 15.3 a $18 \mathrm{t} /$ ha sembradas a densidades de 80 a 90 mil plantas/ha; en híbridos de maíz de origen tropical y templado la producción de materia seca es de 14.5 y 49.7 t/ha en híbridos templados, en híbridos intermedios templados de 13.8 a $14.4 \mathrm{t} / \mathrm{ha}$ y en híbridos intermedios tropical de 14.1 a $14.9 \mathrm{t} / \mathrm{ha}$ de materia seca utilizado para forraje (Nuñes et al., 2001).

CUADRO 3.

Aporte de materia seca del cultivo de maíz al suelo intercalado

\begin{tabular}{|c|c|}
\hline Maiz hibrido $\mathrm{H}-520$ & $\begin{array}{l}\text { Aporte de materia seca al } \\
\text { suelo (kg/ha) }\end{array}$ \\
\hline $\begin{array}{l}\text { 1. Fecha de siembra el } 26 \\
\text { ene } 2018\end{array}$ & $9,250 a b$ \\
\hline $\begin{array}{l}\text { 2. Fecha de siembra el } 09 \\
\text { feb } 2018\end{array}$ & $7,000 \mathrm{~b}$ \\
\hline $\begin{array}{l}\text { 3. Fecha de siembra el } 23 \\
\text { feb } 2018\end{array}$ & $11,333 a$ \\
\hline $\begin{array}{l}\text { 4. Fecha de siembra el } 16 \\
\text { feb } 2019\end{array}$ & $6,667 \mathrm{~b}$ \\
\hline
\end{tabular}

Nota ${ }^{*}$ Letras diferentes representa diferencias estadística significativa al $95 \%$.

Las medias de los tratamientos se encuentran en un rango de aporte de materia orgánica de 1.6 a $3.5 \%$; el suelo de la palma sin aporte de materia seca y el testigo de caña de azúcar tienen una tendencia a presentar bajos contenidos de materia orgánica a una profundidad de 0 a $20 \mathrm{~cm}$. De manera general los arboles manejados con materia seca contienen valores más altos de materia orgánica, solo un caso atípico de árboles de limón persa, manejados sin aporte de materia seca presentan valores similares, a los manejados con materia seca a profundidades de 0 a $20 \mathrm{~cm}$ y de 20 a $40 \mathrm{~cm}$ de profundidad (Figura 6). La materia orgánica, está compuesta 
elementalmente de $\mathrm{C}, \mathrm{H}, \mathrm{O}, \mathrm{N}, \mathrm{P}$ y S en cantidades variables, se considera como una fracción orgánica del suelo, excluidos los residuos animales y vegetales en continuos procesos de descomposición, los residuos de los cultivos aportan alrededor del $40 \mathrm{al} 44 \%$ de carbono al suelo (Galantini y Suñer, 2008).
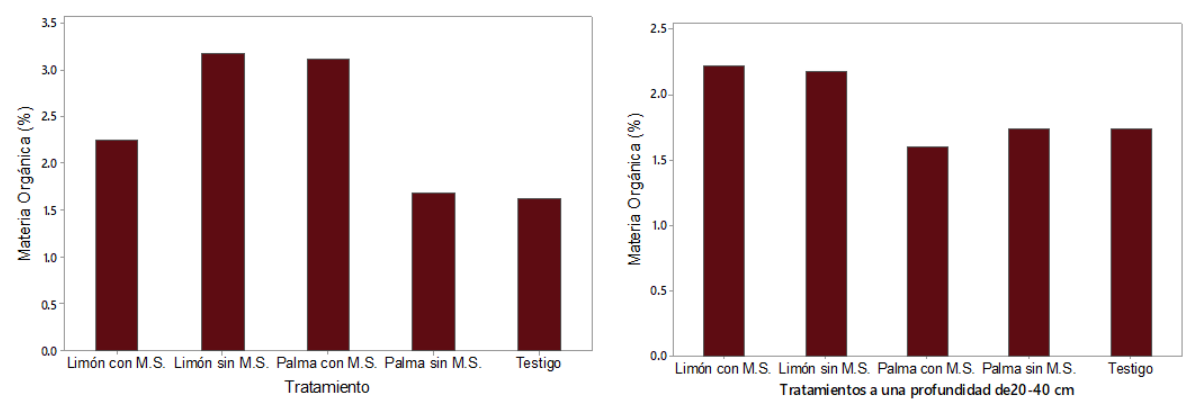

FIGURA 6.

Contenido de materia orgánica en suelo a una profundidad de 0 a 20 y 20

a $40 \mathrm{~cm}$ de profundidad en árboles frutales con y sin aporte de materia seca

En condiciones moderadamente acidas en todos los suelos de las especies de frutales, solamente la palma de coco manejado con materia seca presenta una tendencia a un $\mathrm{pH}$ neutral (Cuadro 4). En arboles de limón persa con buen desarrollo se registra un $\mathrm{pH}$ de 4.5 en suelos de textura arcillosa ligeramente más ácidos a los presentados en el Cuadro 4(Curti et al., 2010). En la palma de coco los rangos de $\mathrm{pH}$ son de 4.4 a 4.7, esta acidificación del suelo es relacionada a las altas aplicaciones de fertilizantes nitrogenados (Teixeira y Da Silva, 2003). El uso de las enmiendas orgánicas mejora las condiciones físico-químicas del suelo, además que en los arboles de cítricos propicia un mejor desarrollo de raíces cuando se utiliza abonos orgánicos al momento de la plantación (Rebolledo et al., 2018).

Los cultivos anuales presentaron menores concentraciones de N-P-K con respecto a los arboles frutales, al comparar la presencia de $\mathrm{NH}_{4}+\mathrm{NO}_{3}$ en suelo a una profundidad de 0 a $20 \mathrm{~cm}$, se encontró que las condiciones son similares. En el contenido de fosforo asimilable de los arboles de limón y palma sin el manejo de materia seca, tienen menores concentraciones al compararlos con los tratamientos con materia seca, este contenido podría deberse a las características nutrimentales de la materia seca aportado al suelo. En el contenido de potasio asimilable los arboles de limón presentaron altas concentraciones con o sin el manejo de materia seca, además que el testigo de limón persa presenta contenidos muy altos de Fe con valores de $122.9 \mathrm{mg} / \mathrm{kg}$ y presencia de Cobre y Zinc en comparación con los demás tratamientos. Los arboles de limón persa manejados con materia seca prefieren sustratos ricos en cobre, calcio y hierro, ya que es requerido para el desarrollo de los arboles, pero no requieren altas concentraciones de magnesio, potasio y zinc, considerando que los arboles de limón persa responden muy bien a la fertilización continua obteniéndose altos rendimientos (Maldonado et al., 2008). Para los arboles de palma de coco, el potasio es necesario para la formación de las nuevas hojas, ya que se presentan altas movilizaciones en el interior de la planta y la escases de este elemento provocaría una deficiencia nutrimental (Teixeira y Da Silva, 2003). Por lo que se recomienda aplicaciones foliares ricos en potasio asperjados al dosel del árbol. 


\section{CUADRO 4.}

Contenido de macro y micro-elementos en suelo de diferentes especies manejadas con y sin materia seca a una profundidad de 0 a $20 \mathrm{~cm}$.

\begin{tabular}{|c|c|c|c|c|c|c|c|c|c|c|}
\hline \multirow{2}{*}{ Tratamientos } & \multirow[t]{2}{*}{$\mathrm{pH}$} & $\mathrm{NH} 4+\mathrm{NO} 3$ & $P$ & $\mathrm{~K}$ & $\mathrm{Ca}$ & $\mathrm{Mg}$ & $\mathrm{Fe}$ & $\mathrm{Cu}$ & $\mathrm{Zn}$ & $\mathrm{Mn}$ \\
\hline & & (\%) & \multicolumn{8}{|c|}{$\mathrm{mg} / \mathrm{kg}$} \\
\hline $\begin{array}{l}\text { 1. Asociación } \\
\text { de frijol y } \\
\text { maiz (NO3) }\end{array}$ & 5.8 & 0.02 & 3.5 & 27 & --- & --- & --- & --- & --- & --- \\
\hline $\begin{array}{l}\text { 2. Limón sin } \\
\text { M.S. }\end{array}$ & 5.4 & 12 & 39.7 & 167 & 1100 & 345 & 122.9 & 0.04 & 0.25 & 4.2 \\
\hline $\begin{array}{l}\text { 3. Limón con } \\
\text { M.S. }\end{array}$ & 6.2 & 9.0 & 47.9 & 121 & 1500 & 318 & 10.1 & + & + & 3.42 \\
\hline $\begin{array}{l}\text { 4. Palma sin } \\
\text { M.S. }\end{array}$ & 6.1 & 9.0 & 25.6 & 43 & 1033 & 265 & 10.91 & + & + & 3.32 \\
\hline $\begin{array}{l}\text { 5. Palma con } \\
\text { M.S. }\end{array}$ & 6.6 & 11 & 32.5 & 30 & 1506 & 337 & 18.48 & + & + & 1.69 \\
\hline $\begin{array}{l}\text { 6. Monocultivo } \\
\text { de caña de } \\
\text { ázucar }\end{array}$ & 5.2 & 11 & 39.7 & 56 & 1567 & 298 & 19.22 & + & + & 9.05 \\
\hline
\end{tabular}

Nota + Trazas, --- valores no analizados.

\section{Conclusiones}

Los árboles de limón persa y coco presentaron un incremento en la altura de planta y diámetro de tallo influenciado por el manejo de materia seca. En efecto los cultivos anuales de maíz y frijol tendieron a presentar incremento en la producción de materia seca, principalmente el cultivo de frijol. Con respecto al análisis físico-químico de suelo se observó que el $\mathrm{pH}$ del suelo manejado con materia seca es ligeramente más altos a los árboles que no se aplicaron materia seca. Existe una buena concentración de materia orgánica superior al monocultivo de caña de azúcar. Los frutales de limón persa empiezan a ensayar la producción. Por lo que se espera que al tercer año de edad ya exista una producción homogénea de todas las especies asociadas.

\section{REFERENCIAS}

Balderas P. F. G. y González A. I. J., 2013. Cocotero híbrido intercalado con cultivos anuales y perennes, tecnología sustentable. Rev. Mex. Cien. For. 4(20): 58-71.

Curti D. S. A., Hernández G. C., Loredo S. X., 2012. Productividad del limón persa injertado en cuatro portainjertos en una huerta comercial de Veracruz, México. Revista Chapingo Serie Horticultura 18(3): 291-305

Duarte M. J., Pezo D. A., Arze J., 1994. Crecimiento de tres gramíneas forrajeras establecidas en un cultivo intercalado con maíz (Zea mays L.) o vigna (vigna unguiculata). Pasturas tropicales. 16(1): 8-14.

Fernández J. L., Benítez D. E., Gómez I., De Souza A. Espinoza R., 2004. Rendimiento de materia seca y contenido de proteína bruta del pasto Panicum máximum vc likoni en un suelo vertisol de la provincia Granma. Revista Cubana Agrícola, 38(4): 417-422.

Galantini J. A., y Suñer L., 2008. Las fracciones orgánicas del suelo: análisis en los suelos de la Argentina. Agriscientia, 25(1): 41-55.

Godoy M. L., Díaz C. G., Vásconez M. G., Defaz D. E. y González O. B., 2011. Evaluación de dos variedades de frijol durante tres épocas de siembra bajo sistema de cultivo asociado con Maíz. Ciencia y Tecnología. 4(1):5-11. https://doi.org/10.18779/cyt.v4i1.50 
Granados S. D., López-Ríos G. F., 2002. Manejo de la palma de coco (Cocos nucifera L.) en México. Revista chapingo serie ciencias forestales y del ambiente. 8(1):39-48.

Hernández Z. R. D., Flores C. R. J., Isiordia A. N., Robles B. A., López M. G., Sotelo M. A, M., 2017. Temperatura y humedad relativa en poblaciones de ácaros fitófagos asociados alc cultivo de limón (Citrus limón Burn) en Xalisco, Nayarit. Entomología mexicana 4:8-14.

Leguía H., Pietrarelli L., Luque. S. M., Sánchez J., Alessandría E., Arborno M. y Zamar J. L., 2004. El bosque nativo como referente del deterioro de los suelos agrícolas. Revista de agroecología, 19(4): 28-31

Maldonado T. R., Almaguer V. G., Álvarez S. M. E. y Robledo S. E., 2008. Diagnóstico nutrimental y validación de dosis de fertilización para limón persa. Terra Latinoamericana 26 (4):341-349.

Matias, S. S. R., Aquino, B.F., Freitas, J. A. D., Camacho-Tamayo, J. H. 2008. Efecto de la fertirrigación con n y k en el crecimiento de la palma de coco enano Verde de jiqui. Bioagro [online]. 20(3): 177-183.

Megchún G. J. V., 2007. Chile piquín (Capsicum annum var. Aviculare) intercalado en guanábana (Annona muricata L.) en diferentes densidades de siembra y cubierta plástica. Dirección general de educación superior tecnológica. ITUG. 116p.

Mercado R. G., 2016. La Bioeconomía-concepto y aplicación al desarrollo rural. RIIARn, 3(2): 188-193.

Nuñes H. G., Faz C. R., Tovar G. M. del R., Zavala G. A., 2001. Híbridos de maíz para la producción de forraje con alta digestibilidad en el norte de México. Técnica Pecuaria en México. 39(2): 77-78.

Ortega E. A., Álvarez M. A., Del Valle M. del C., Chauvete M., 2005. La economía de los sistemas campesinos de producción de leche en el estado de México. Técnica Pecuaria en México. 43 (1): 39-56.

Paz R., Jara C., Nazar P., 2013. Economía Social y Agricultura Familiar. La experiencia de la Feria de Villa Río Hondo (Argentina). Revista Venezolana de Economía Social. 13 (25): 53-74.

Rebolledo M. L., Megchún G. J. V., Rebolledo M. A. y Rodríguez L. D. A., 2018. Desarrollo de plantas de coco y limón persa con aporte de materia seca por cultivos intercalados. Avances en investigación agrícola, pecuaria, forestal, acuícola, pesquería, desarrollo rural, transferencia de tecnología, biotecnología, ambiente, recursos naturales y cambio climático. INIFAP, CP, UACH, INAPESCA, UV, TECNM. Medellín Veracruz, México. 2(1): 368-378.

Teixeira L. A. J., Da Silva A. A., 2003. Nutricao mineral de populacoes e hibridos de coqueiro (Cocos nucifera L.) cultivados em bebedouro (SP). Rev. Bras. Frutic. Jaboticabal. Sp. 25(2): 371-374. 